\title{
Free partial patch and partial tube jejunal graft transfers were used to reconstruct pharyngoesophageal defect: Our experience with three cases
}

\author{
Jifeng Liu ${ }^{1}$, Rong $\mathrm{Yu}^{1}$, Di Deng ${ }^{1}$, Linke $\mathrm{Li}^{1}$, Ji Wang ${ }^{1}$, Bo $\mathrm{Li}^{1}$, Dan $\mathrm{Lv}^{1}$, Jun Liu ${ }^{1}$, and Fei \\ Chen $^{1}$ \\ ${ }^{1}$ Sichuan University West China Hospital
}

April 15, 2021

\begin{abstract}
Those patients with hypopharyngoesophageal cancer often sacrificed larynx before reconstruction using jejunum to restore the continuity of the digestive tract and allow oral alimentation. We retrospectively collected and analyzed three patients who underwent hypopharyngoesophageal reconstruction by free partial patch and partial tube jejunal graft transfer with reservation of laryngeal function caused by hypopharyngeal cancer invading the cervical esophagus. The partial patch and partial tube jejunal graft transfer survival rate was $100 \%(3 / 3)$. The larynx was reserved in the three patients. The partial patch and partial tube jejunal graft transfer is a safe and reliable choice for reconstruction of large and complex defects after pharyngectomy and cervical esophagectomy with larynx preserved.
\end{abstract}

Title: Free partial patch and partial tube jejunal graft transfers were used to reconstruct pharyngoesophageal defect: Our experience with three cases

Running Title: Free partial patch and partial tube jejunal graft transfers reconstruct pharyngoesophageal defect

Key points: Those patients with hypopharyngoesophageal cancer often sacrificed larynx before reconstruction using jejunum to restore the continuity of the digestive tract and allow oral alimentation. We retrospectively collected and analyzed three patients who underwent hypopharyngoesophageal reconstruction by free partial patch and partial tube jejunal graft transfer with reservation of laryngeal function caused by hypopharyngeal cancer invading the cervical esophagus. The partial patch and partial tube jejunal graft transfer survival rate was $100 \%(3 / 3)$. The larynx was reserved in the three patients. The partial patch and partial tube jejunal graft transfer is a safe and reliable choice for reconstruction of large and complex defects after pharyngectomy and cervical esophagectomy with larynx preserved.

\section{INTRODUCTION}

The revascularized free jejunum has been widely used to reconstruct the hypopharyngoesophageal tract[1]. Most of those patients sacrificed larynx before reconstruction using jejunum to restore the continuity of the digestive tract and allow oral alimentation. To fit the complex defect and preserve laryngeal function, we longitudinally incised jejunum partially to form partial patches and partial tubes graft transfer, and reconstruct the large or even circumferential hypopharyngoesophageal defect in patients with squamous cell carcinoma of the hypopharynx (SCCHP) invading the cervical esophagus. This might help to enlarge indications of jejunum in reconstruction pharyngoesophageal with preservation of larynx. 


\section{METHODS}

Three male patients with advanced SCCHP invading cervical esophageal without partial response on two cycle of induction chemotherapy[Cisplatin (DDP) +5 -fluorouracil $(5$-FU)] were collected in $<$ Blinded for review $>$. The preoperatively clinical characteristics and stage (International Union Against Cancer, 2010) of the three patients were listed in Table 1. All the patients were diagnosed with SCCHP by histopathological examination in our hospital. Then we conducted radical tumor resection followed by chemoradiotherapy for these patients. The present study was approved by the Ethics Committee of $<$ Blinded for review $>$.

Bilateral neck selective dissection, partial cervical esophagectomy and tumor resection with an $1 \mathrm{~cm}$ safe margin was performed in the three patients (Figure 1a).Partial hypopharyngectomy was conducted in $2 / 3$ patients, total hypopharyngectomy in $1 / 3$ patients; partial laryngectomy in $1 / 3$ patients. Three patients' larynx was partially or completely preserved. Meanwhile, the recipient vessels and superior and recurrent laryngeal nerves were identified and preserved.

A free jejunal graft transfer(FJGT) was harvested and partially incised in longitudinal axis along the antimesenteric border to gain a FJGTs with partial patch and tube (Figure $1 \mathrm{~b}$ and c). The FJGTs were transferred to the defect site and sutured to the residual edge of pharynx, esophagus and larynx(Figure 1 d). Then the vessels anastomosis was conducted.

To monitor survival status of the FJGTs, a "mini watch jejunum" was made and kept outside of the neck incision (Figure 1d). After wound recovery, all patients received adjuvant radiotherapy and $2 / 3$ of them also received chemotherapy(two-cycles of DDP +5 -FU).

The operative complications, the function of pharynx, esophagus and larynx reconstructed were evaluated. Also the survival state of patients were collected.

\section{RESULTS}

Postoperative information was listed in Table 2.

The three FJGTs were all survival after surgery(Figure 2). One patient had a subcutaneous infection. With treatments (e.g.drainage, dressing changes, systemic antibiotic treatment and nutritional support), the patient recovered within four weeks. None of the three patients had donor site complications.

The time of oral feeding after operation ranged from 21 to 40 days, and hospital day from 14 to 35 days. All the patients were tracheostomy dependent after operation, and the duration time from surgery to decannulation ranged from 4 months to 5 months.

Two patients could communicate with others freely after treatment. Although one patient's speech function was affected, he still could communicate with others by speech. The three patients had normal swallowing function.

The follow-up time ranged from 52 to 74 months. Two patients survived without tumor relapse and metastasis when data were collected. One patient suffered from lymph node metastasis of the posterior pharyngeal space 36 months after treatment (Table 2).A second surgery and followed chemoradiotherapy was conducted for the patient. Interestingly, the jejunal graft transfer still functioned.

\section{DISCUSSION}

Induction chemotherapy followed by radiotherapy or synchronous chemoradiation have been the main treatment for SCCHP patients [2]. In our cases, the tumors were not controlled by induction chemotherapy. For those patients, radical surgery usually include total laryngopharyngectomy and cervical esophageal resection[3], which would lead to huge and complex defect in the boundary between respiration and digestive tract. As a great challenge, even some cases without larynx invasion, the total laryngectomy was performed to facility the defect reconstruction [4]. 
Jejunum is a reconstructive choice for the hypopharynx and cervical esophagus defect. As a tube, jejunum used to recover the continuity of hypopharynx and cervical esophagus often need to remove larynx. Nakatsuka and Miyamoto et al.[5] had used jejunal patch to reconstruct the defect of hypopharynx with laryngeal preservation. However, whether the jejunal tube or patch alone, it is still great challenge for surgeons to repair the complex defect of hypopharynx with larynx preservation, especially when the cervical esophagus was invaded. To preserve the larynx, we incised jejunum partially along the longitudinal axis to gain a partial patch and tube FJGTs other than previous tube or patch shape alone, so that the FJGTs could be suitable for the complex defect owing to the preservation of larynx.

In our cases, all the FJGTs survived, even after radiation, which indicated that partially longitudinal incision of free jejunum did not increase the risk of necrosis of FJGTs, even the partial patch and tube FJGTs can be tolerance to radiotherapy. Importantly, all of them obtained the preservation of larynx presented with tracheostomy independency and speech function. Only one patients' speech function was weakened. However, he still could communicate with others by speech. At least, he could avoid tracheostomy independency. One patients suffered from posterior pharyngeal space lymph node metastasis after the first operation. The transplant jejunum still survived after lymph node dissection followed by the second radical chemoradiotherapy.

\section{CONCLUSIONS}

Our study indicated that the free jejunum with partial incision along the antimesenteric border might be a potential option to reconstruct the complex hypopharyngoesophageal defects with the preservation of laryngeal function for patients with advanced SCCHP.

Consent for publication: Any information which might identify the patients were strictly removed before data analyses. Written informed consent was obtained from all the six patients for the presentation.

\section{References}

1. Seidenberg B. Immediate reconstruction of the cervical oesophagus by vascularised isolated jejunal segment. Annals of Surgery. 1959; 149.

2. Habib A. Management of advanced hypopharyngeal carcinoma: systematic review of survival following surgical and non-surgical treatments. The Journal of laryngology and otology. 2018; 132(5):385-400.

3. Bova R, Goh R, Poulson M, Coman WB. Total pharyngolaryngectomy for squamous cell carcinoma of the hypopharynx: a review. The Laryngoscope. 2005; 115(5):864-9.

4. Chu PY, Chang S-Y. Reconstruction of the Hypopharynx After Surgical Treatment of Squamous Cell Carcinoma. Journal of the Chinese Medical Association. ; 72(7):351-5.

5. Miyamoto S, Sakuraba M, Asano T, Hayashi R, Ebihara M, Miyazaki M, Daiko H, Shinozaki T, Kimata Y. Free jejunal patch graft for reconstruction after partial hypopharyngectomy with laryngeal preservation. Arch Otolaryngol Head Neck Surg. 2011; 137(2):181-6.

Figure.1. a, The defect of cervical esophagus (yellow arrow) and larynx (blue arrow); b, Harvesting of free jejunal flap; c, The free jejunal flaps were partially longitudinally dissected; d Pharynx and esophagus reconstruction.

Figure.2. Postoperative laryngoscopy of the reconstructed pharynx and esophagus (red arrow shows jejunal graft transfer ). 

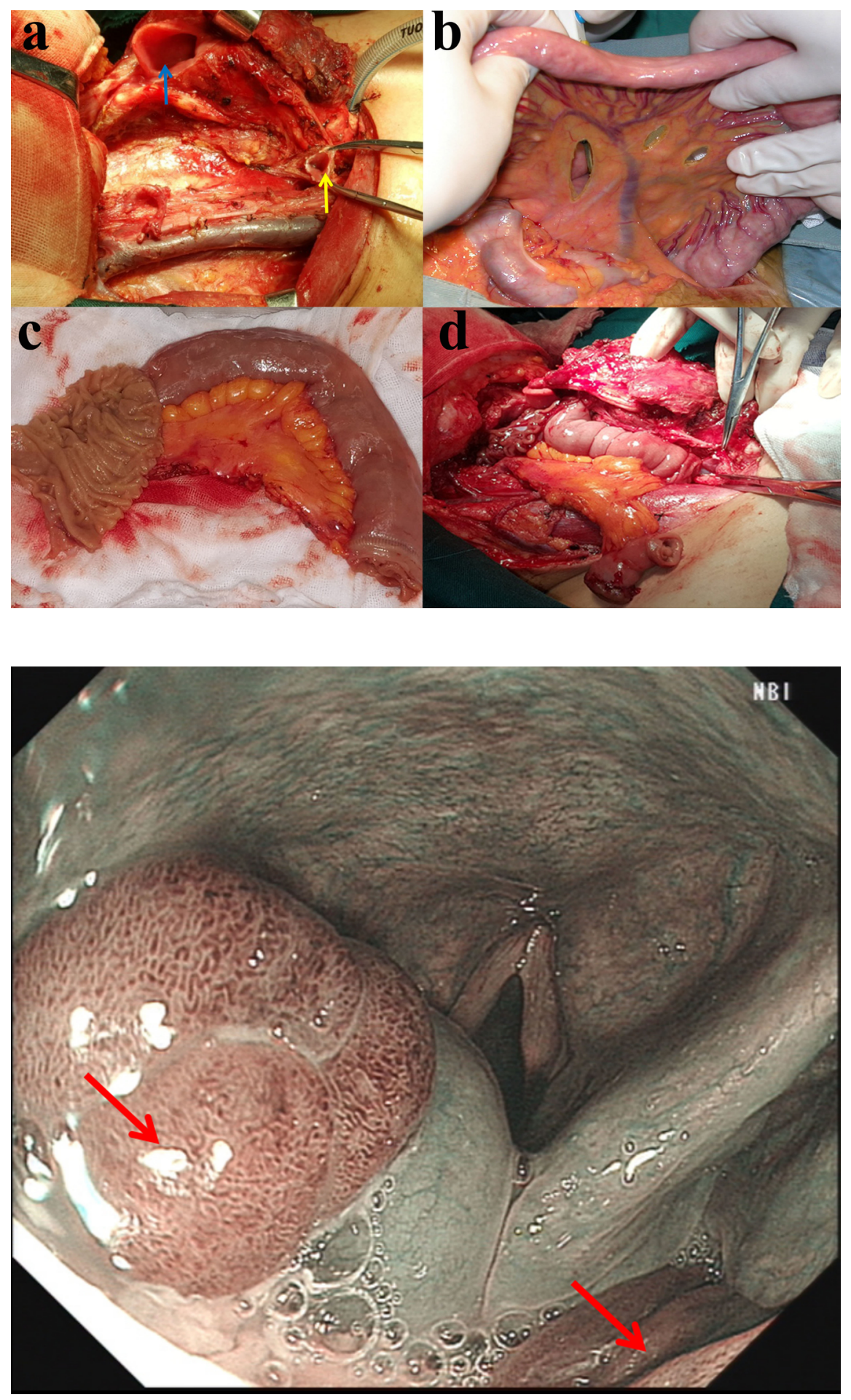

\section{Hosted file}

Table 1 Patient demographic data.pdf available at https://authorea.com/users/407984/ articles/518143-free-partial-patch-and-partial-tube-jejunal-graft-transfers-were-usedto-reconstruct-pharyngoesophageal-defect-our-experience-with-three-cases 


\section{Hosted file}

Table 2 Postoperative information.pdf available at https://authorea.com/users/407984/ articles/518143-free-partial-patch-and-partial-tube-jejunal-graft-transfers-were-usedto-reconstruct-pharyngoesophageal-defect-our-experience-with-three-cases 